\title{
GESTÃO DE ESTOQUES HUMANITÁRIOS UTILIZANDO SIMULAÇÃO DE MONTE CARLO
}

\author{
Daniel Eckhardt \\ Pontifícia Universidade Católica do Rio de Janeiro \\ Rua Marquês de São Vicente, 225, Gávea - Rio de Janeiro, RJ - Brasil \\ daneckhardt@gmail.com \\ Brenda de Farias Oliveira Cardoso \\ Pontifícia Universidade Católica do Rio de Janeiro \\ Rua Marquês de São Vicente, 225, Gávea - Rio de Janeiro, RJ - Brasil \\ brendafarias.eng@outlook.com \\ Adriana Leiras \\ Pontifícia Universidade Católica do Rio de Janeiro \\ Rua Marquês de São Vicente, 225, Gávea - Rio de Janeiro, RJ - Brasil \\ adrianaleiras@puc-rio.br
}

\section{RESUMO}

A importância da logística humanitária recai sobre o significativo número de desastres registrados ao longo do tempo, pelo volume financeiro transacionado mundialmente e principalmente pelos danos e perdas causados. $\mathrm{O}$ aprimoramento das técnicas de gestão dos recursos para garantir o sucesso de uma resposta efetiva a um desastre é um grande desafio. Neste contexto, este artigo objetiva propor um instrumento de controle de estoque que considera um índice (disponibilidade de recursos humanitários) de atendimento adequado aos beneficiários de um desastre através de simulação de Monte Carlo. Além disso, são utilizados dados do desastre ocorrido na região serrana do Rio de Janeiro em 2011, entrevistas com especialistas e uma análise de sensibilidade nos parâmetros Estoque Mínimo e Lote Padrão, de forma a aumentar a demanda atendida. Como principal resultado, conclui-se que ajustes referentes aos parâmetros Estoque Mínimo e Lote Padrão geram alterações significativas nos níveis de estoque e atendimento da demanda que, por sua vez, estão diretamente relacionados com a demanda e com fatores externos.

Palavra-chave: Logística Humanitária; Simulação; Gestão de Estoque.

\begin{abstract}
The importance of humanitarian logistics lies with the significant number of disasters reported over the past decades, the financial volume traded, and mainly due to the damages and losses generated by disasters. The improvement of resource management techniques to ensure the success of an effective response to a disaster is a major challenge. In this context, this paper aims to provide an inventory control tool that considers an index (availability of humanitarian resources) to adequately attend the beneficiaries of a disaster
\end{abstract}


through Monte Carlo simulation. Besides, data from the disaster occurring in the mountain region of Rio de Janeiro in 2011, interviews with experts and a sensitivity analysis on the Minimum Stock and Standard Lot parameters are used, in order to increase the demand. As a main result, it can be concluded that adjustments related to the Minimum Stock and Standard Lot parameters generate significant changes in the levels of inventory and demand, which in turn are directly related to demand and external factors.

Keywords: Humanitarian Logistics; Simulation; Inventory Management.

\section{Como Citar:}

ECKHARDT, Daniel; CARDOSO, Brenda de Farias Oliveira; LEIRAS, Adriana. Gestão de Estoques Humanitários utilizando Simulação de Monte Carlo. In: SIMPÓSIO DE PESQUISA OPERACIONAL E LOGÍSTICA DA MARINHA, 19., 2019, Rio de Janeiro, RJ. Anais [...]. Rio de Janeiro: Centro de Análises de Sistemas Navais, 2019.

\section{INTRODUÇÃO}

A Logística Humanitária (LH) pode ser definida como o processo de planejar, implementar e controlar as atividades referentes preparação, planejamento, aquisição, transporte, armazenagem, rastreamento e desembaraços alfandegários dos recursos disponíveis, desde o ponto de origem até o ponto de consumo, a fim de atender as necessidades dos beneficiários, neste caso, as pessoas afetadas por desastres naturais, por exemplo, tsunamis, enchentes, terremotos, ou por desastres causados pelo homem, por exemplo, desabamentos e explosões nucleares (THOMAS e KOPCZAK, 2005).

Os desastres, por sua vez, são problemas complexos, com alto grau de incerteza nas informações e com limitadores extremos de recursos (humanos e materiais), em ambientes severos e dinâmicos (ÇELIK et al., 2012). Esses desastres são divididos em súbitos (terremotos, tsunamis, ataques terroristas) e lentos (fome, pobreza ou seca extrema) e são caracterizados por quatro fases principais: mitigação, preparação, resposta e reabilitação ou reconstrução (VAN WASSENHOVE, 2006).

Eckhardt e Leiras (2018) descrevem estas fases: (i) mitigação: abrange atividades, projetos ou ações que visam impedir ou reduzir os impactos de um desastre; (ii) preparação: envolve as atividades possíveis de serem realizadas para uma resposta antes que o desastre ocorra; (ii) resposta: fase reativa, tendo em vista que as entidades, o governo e a população atuam diretamente no salvamento de vidas e na preservação dos recursos humanos e financeiros da região afetada; e reconstrução: foca no restabelecimento financeiro, social e patrimonial da região afetada.

Ozbay e Ozguven (2007) apresentam três questões básicas a serem consideradas durante e/ou depois de um desastre: (i) configurar centros de distribuição que armazenem os suprimentos necessários para serem recebidos e distribuídos; (ii) instalar centros de distribuição e armazenamento com gestão de inventário automatizado capaz de realizar uma eficiente distribuição de suprimentos; e (iii) implementar um processo de rotatividade de estoques inovador que assegure que os suprimentos não estraguem. Em relação ao préposicionamento de suprimentos, Beamon e Kotebla (2006) afirmam que é uma estratégia de armazenagem normalmente vista em respostas de emergência humanitárias complexas. As decisões sobre localização afetam o desempenho das operações emergenciais de assistência, uma vez que o número e a localização dos centros de distribuição bem como o total de recursos, afetam diretamente o tempo de resposta e os custos observados ao longo da cadeia de suprimentos (BARBOSA et al., 2010). 
Beamin e Kotleba (2006) citam três estratégias possíveis para gestão de estoques humanitários: (i) modelos matemáticos, sistema de avaliação contínua de inventário; (ii) modelos heurísticos, os quais fornecem bons (não ideal) resultados; e (iii) modelos ingênuos, simples e fáceis de implementar, requerendo mínimo esforço computacional.

Neste contexto, o objetivo principal deste artigo é verificar a aplicação da simulação de Monte Carlo, em um modelo, de forma a proporcionar um instrumento de controle de estoque que evidencie um índice (disponibilidade de recursos humanitários) de atendimento adequado aos beneficiários. Ainda, neste artigo são utilizados dados do desastre ocorrido na região serrana do Rio de Janeiro em 2011, entrevistas com especialistas e uma análise de sensibilidade nos parâmetros Estoque Mínimo e Lote Padrão, de forma a aumentar a demanda atendida.

Este artigo está dividido em cinco seções, incluindo esta introdução. A Seção 2 apresenta a metodologia utilizada. A Seção 3 apresenta a revisão da literatura. Na Seção 4, é apresentado o modelo matemático. A Seção 5 apresenta a análise de sensibilidade. A Seção 6 apresenta as considerações finais. E a Seção 7 apresenta as referências utilizadas no trabalho.

\section{METODOLOGIA DA PESQUISA}

A pesquisa proposta neste artigo pode ser classificada em descritiva e metodológica. De acordo com Vergara (2014) uma pesquisa descritiva expõe características de determinada população ou de determinado fenômeno, não tendo compromisso de explicar os fenômenos que descreve, embora sirva de base para tal explicação. A mesma autora define a pesquisa metodológica como o estudo que se refere a instrumentos de captação ou de manipulação da realidade - está, portanto, associado a caminhos, formas, maneiras, procedimentos para atingir determinado fim.

Para alcançar o objetivo deste estudo, são utilizadas quatro etapas principais: (i) fundamentação teórica; (ii) entrevistas; (iii) proposta do modelo matemático; e (iv) análise de sensibilidade. A Figura 1 mostra o diagrama das etapas no contexto deste estudo.

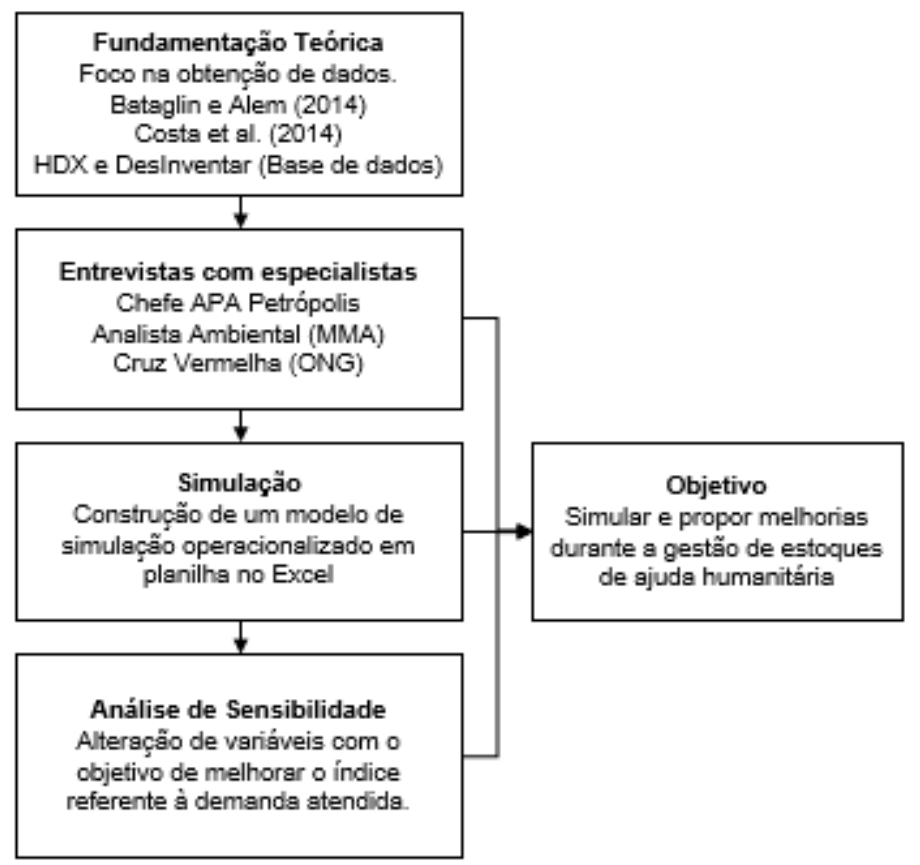

Figura 1: Quadro esquemático (metodologia)

Fonte: Autores (2019) 
A fundamentação teórica objetiva levantar as publicações relacionadas à modelagem matemática, no contexto de LH para contextualizar e conceituar de forma qualitativa e quantitativa temas relacionados com apoio a decisão em gestão de estoques em desastres e simulação.

As entrevistas, realizadas com três especialistas em LH, possui o objetivo de validar os resultados obtidos por meio da pesquisa bibliográfica e identificar possíveis contribuições para o estudo apresentado, como revisão e validação dos dados de entradas.

A metodologia de simulação segue o modelo proposto por Chwif (1999), que consiste no sequenciamento dos seguintes processos: (i) entender claramente o sistema a ser simulado e os seus objetivos; (ii) representar o modelo de forma abstrata (através de diagramas, por exemplo); (iii) definir o modelo conceitual (nesta etapa os dados de entrada já devem ter sido mapeados); (iv) criar o modelo computacional (neste artigo foram utilizados os seguintes softwares @Risk, Excel e XLSTAT); (v) operacionalizar e realizar experimentos, nesta etapa são efetuadas várias "rodadas" do modelo e os resultados da simulação são analisados.

Conforme contextualizado na introdução deste artigo, o alto grau de incerteza é uma das dificuldades nas operações humanitárias e isso inclui a incerteza na demanda. Entretanto, por meio da revisão da literatura e entrevistas com especialistas em desastres, estima-se um possível comportamento da demanda para um desastre específico, neste caso, o desastre da Região Serrana do Rio de Janeiro, ocorrido em 2011.

A partir de dados estimados de demanda de kits de emergência (alimentos, medicamentos, água) propostos por Bataglin e Alem (2014), é realizado um teste Augmented Dickey-Fuller (ADF), utilizando o software XLSTAT, para verificar a estacionariedade das curvas de demanda - com objetivo de validar se as séries propostas tinham alguma tendência ou temporalidade.

Com suporte do software @Risk, as distribuições de probabilidade são ajustadas para as três demandas analisadas. O resultado destes ajustes possibilita a geração de dados aleatórios para simular o comportamento da demanda destes itens. Por fim, a etapa de Análise de Sensibilidade consiste em verificar o efeito da a variação do 'Estoque Mínimo' e 'Lote Padrão' com objetivo de otimizar o nível de atendimento aos beneficiários.

\section{SIMULAÇÃO NO CONTEXTO HUMANITÁRIO}

Modelos matemáticos têm sido utilizados com objetivo de otimizar, contornar e até solucionar os problemas citados anteriormente. Para Charles e Lauras (2011), o número de artigos científicos publicados cresceu consideravelmente nos últimos anos, sendo que algumas publicações utilizam as técnicas quantitativas, como otimização e simulação para suporte à tomada de decisões gerenciais.

De acordo com Leiras et al. (2014), pesquisas quantitativas relacionadas ao problema de roteamento de veículo são mais comuns. Esta informação justifica-se uma vez que roteirização é considerada o segundo maior custo operacional das organizações humanitárias, perdendo somente para despesas com pessoas (VAN WASSENHOVE et al., 2011)

Já no campo da gestão de estoque, embora haja uma literatura bem estabelecida abordando as questões básicas de inventário, por exemplo, quando comprar e onde estocar, os modelos e políticas existentes são pouco aplicados ao gerenciamento de inventário humanitário (BALCIK et al., 2016). A Tabela 1 apresenta alguns artigos, extraídos da base de dados Scopus, que utilizam técnicas de simulação relacionadas com a gestão de estoques no contexto de logística humanitária. São artigos de periódicos e conferências e, somente os que estão inseridos no cenário humanitário, são analisados para fundamentação teórica. 
Tabela 1: Referências bibliográficas de simulação no contexto de LH

\begin{tabular}{|c|c|}
\hline Autores & Descrição \\
\hline $\begin{array}{l}\text { Han e Wei } \\
\text { (2018) }\end{array}$ & $\begin{array}{l}\text { Construção de um modelo para cadeia de suprimentos de emergência, combinando as } \\
\text { estratégias de transbordo lateral, reabastecimento de emergência e CONWIP (Constant } \\
\text { Work in Process), onde simulações de dinâmica do sistema são usadas para testar a } \\
\text { eficácia das estratégias propostas. }\end{array}$ \\
\hline$\frac{\text { Toyasaki et al. }}{(2016)}$ & $\begin{array}{l}\text { Abordagem de pesquisa baseada em dados empíricos e modelagem quantitativa para } \\
\text { analisar e superar os desafios da coordenaçao de rede, baseando-se em um modelo de } \\
\text { jornaleiro. A criação da rede aborda a cooperação horizontal de organizaçõe na gestão de } \\
\text { estoques e um mecanismo de coordenação que alcança a otimização do sistema e gera } \\
\text { impactos nas de racionamento de estoque. Propõe-se também uma política de prioridade } \\
\text { para o gerenciamento do inventário. }\end{array}$ \\
\hline $\begin{array}{l}\text { Garrido et al. } \\
\text { (2015) }\end{array}$ & $\begin{array}{l}\text { Apresentação de um modelo para auxiliar os tomadores de decisão na logística de uma } \\
\text { emergência de inundação. O modelo tenta otimizar os níveis de estoque para suprimentos } \\
\text { de emergência, bem como a disponibilidade dos veículos, a fim de fornecer suprimentos } \\
\text { suficientes para atender às demandas com uma determinada probabilidade. }\end{array}$ \\
\hline $\begin{array}{l}\text { Mulyono e } \\
\text { Ishida (2014) }\end{array}$ & $\begin{array}{l}\text { Proposta de um método para melhorar o desempenho das operações de transbordo lateral } \\
\text { através da formação de clusters de abrigos antes que ocorra o desastre. O modelo é valida- } \\
\text { do com técnica de simulação e os resultados mostram que a formação de clusters contribui } \\
\text { para um melhor desempenho de logística e inventário. }\end{array}$ \\
\hline $\begin{array}{l}\text { Campbell e } \\
\text { Jones (2011) }\end{array}$ & $\begin{array}{l}\text { Apresentação de equações de estoque ideal e custo toal de entrega para decisões sobre } \\
\text { onde preposicionar suprimentos em preparação para um desastre e quanto a preposição em } \\
\text { um local, levando em consideração o risco dos suprimentos estarem mais próximos de } \\
\text { possíveis locais de desastres. }\end{array}$ \\
\hline Lodree (2011) & $\begin{array}{l}\text { Apresentação de políticas de controle de estoque pró-ativo e reativo no contexto do prová- } \\
\text { vel surto de demanda de pré-tempestade para um item de suprimento de emergência que } \\
\text { se move rapidamente e identificam as condições mais condizentes com cada estratégia de } \\
\text { acordo com o critério de decisão minimax. }\end{array}$ \\
\hline $\begin{array}{l}\text { McCoy e } \\
\text { Brandeau } \\
(2011)\end{array}$ & $\begin{array}{l}\text { Desenvolvimento um modelo de inventário para analisar a interação entre um estoque e } \\
\text { um campo de refugiados a jusante ou operação de socorro, considerando duas decisões de } \\
\text { inventário: particionamento de um orçamento fixo entre os custos de estocagem e expedi- } \\
\text { ção; e transporte itens de alívio do estoque para uma operação de alívio a jusante de uma } \\
\text { maneira eficiente. }\end{array}$ \\
\hline $\begin{array}{l}\text { Lee et al. } \\
\text { (2006) }\end{array}$ & $\begin{array}{l}\text { Descrição de um sistema de simulação e suporte à decisão para o planejamento de clínicas } \\
\text { de dispensação de emergência em grande escala para responder a ameaças biológicas e a } \\
\text { surtos de doenças infecciosas. }\end{array}$ \\
\hline $\begin{array}{l}\text { Aaby et al. } \\
\text { (2006) }\end{array}$ & $\begin{array}{l}\text { Desenvolvimento de modelos para melhorar o planejamento clínico dos Serviços de Saúde } \\
\text { Pública do Condado de Montgomery (Maryland), que incluem modelos de simulação de } \\
\text { eventos discretos e modelos de planejamento de capacidade e de sistema de filas. }\end{array}$ \\
\hline $\begin{array}{c}\text { Beamon e } \\
\text { Kotleba (2006) }\end{array}$ & $\begin{array}{l}\text { Desenvolvimento e teste de três estratégias diferentes (modelo matemático, modelo } \\
\text { heurístico e modelo ingênuo) de gerenciamento de estoque aplicadas à emergência } \\
\text { complexa no sul do Sudão. Identificação de fatores críticos do sistema que contribuíram } \\
\text { mais significativamente para o desempenho do sistema de inventário e pontos fortes e } \\
\text { fracos de cada estratégia de gerenciamento de estoque. }\end{array}$ \\
\hline $\begin{array}{c}\text { Taskin e } \\
\text { Lodree (2010) }\end{array}$ & $\begin{array}{l}\text { Apresentação de um problema estocástico de controle de estoque para empresas de manu- } \\
\text { fatura e varejo que enfrentam decisões desafiadoras de aquisição e produção associadas às } \\
\text { temporadas de furacões, por meio de um problema de controle de estoques multi-período. }\end{array}$ \\
\hline
\end{tabular}

Fonte: Autores (2019)

Dessa maneira, ainda há a necessidade de estudos utilizando técnicas quantitativas como, por exemplo, técnicas de simulação, no auxílio de problemas relacionados com logística humanitária. A decisão de estocar ou comprar após o desastre e a determinação do ponto de pedido ideal correspondem a maior parte dos estudos listados. Para todos os autores, a falta de dados precisos e as incertezas dos eventos são os maiores desafios para melhorar as técnicas de gestão de estoques. 


\section{DESCRIÇÃO DO PROBLEMA E MODELO PROPOSTO}

De acordo com Bandeira et al. (2011), o desastre natural ocorrido em 2011, em 20 cidades do Rio de Janeiro é considerado o maior desastre natural ocorrido no Brasil, onde 916 pessoas morreram e 90.000 foram diretamente afetadas. As causas desta tempestade, descritas pelos autores, foram: a geologia da região, a ocupação irregular (em encostas e planícies aluviais) e precipitação intensa (em períodos de 15 minutos).

A fase de resposta ao desastre apresentou vários problemas, descritos por Bandeira et al. (2011): problemas de logística devido rodovias e estradas interrompidas; baixo nível de planejamento e eficiência na utilização dos recursos disponíveis (por exemplo, helicópteros parados no campo); falta de kits de ajuda (médicos, alimentos e água); remoção de cadáveres; problemas na distribuição de ajuda (por exemplo, nenhum planejamento para alocação de kits médicos e medicamentos). Além desses problemas, Costa et al. (2014) identificaram: falta de informações sobre as dimensões reais da catástrofe; saques e insegurança em alguns locais afetados; falta de transporte adequado; dificuldades na utilização do sistema de comunicação disponível devido a topografia da região; má qualidade dos mapas disponíveis.

Costa et al. (2014) destacam ainda que a distribuição das doações era para ser realizada pelas Forças Armadas, no entanto, devido à falta de informações, a dificuldade de comunicação de rádio e pessoal de campo pouco qualificados, a distribuição dos alimentos foi realizada de forma quase aleatória gerando problemas, por exemplo, falta de combustível e da própria distribuição dos alimentos.

Devido à falta de registro de informações (demandas, número de pessoas afetadas, entidades de ajuda, quantidade de doações) após o desastre ocorrido no Rio de Janeiro, as fontes de dados utilizadas neste artigo são baseadas no estudo apresentado por Bataglin e Alem (2014) que calculam a demanda necessária de recursos (kits de sobrevivência) a partir do número total de desalojados, desabrigados e mortos. $\mathrm{O}$ estudo realizado pelos autores foi baseado no Projeto Esfera (Sphere Handbook, 2011), que consiste em normas mínimas (quantidade de água, alimentos, itens não alimentares) que devem ser seguidas para uma resposta humanitária efetiva. A Figura 2 apresenta as curvas de demanda para os três kits apresentados por Bataglin e Alem (2014): kits alimentares, kits de água e kits de medicamentos.

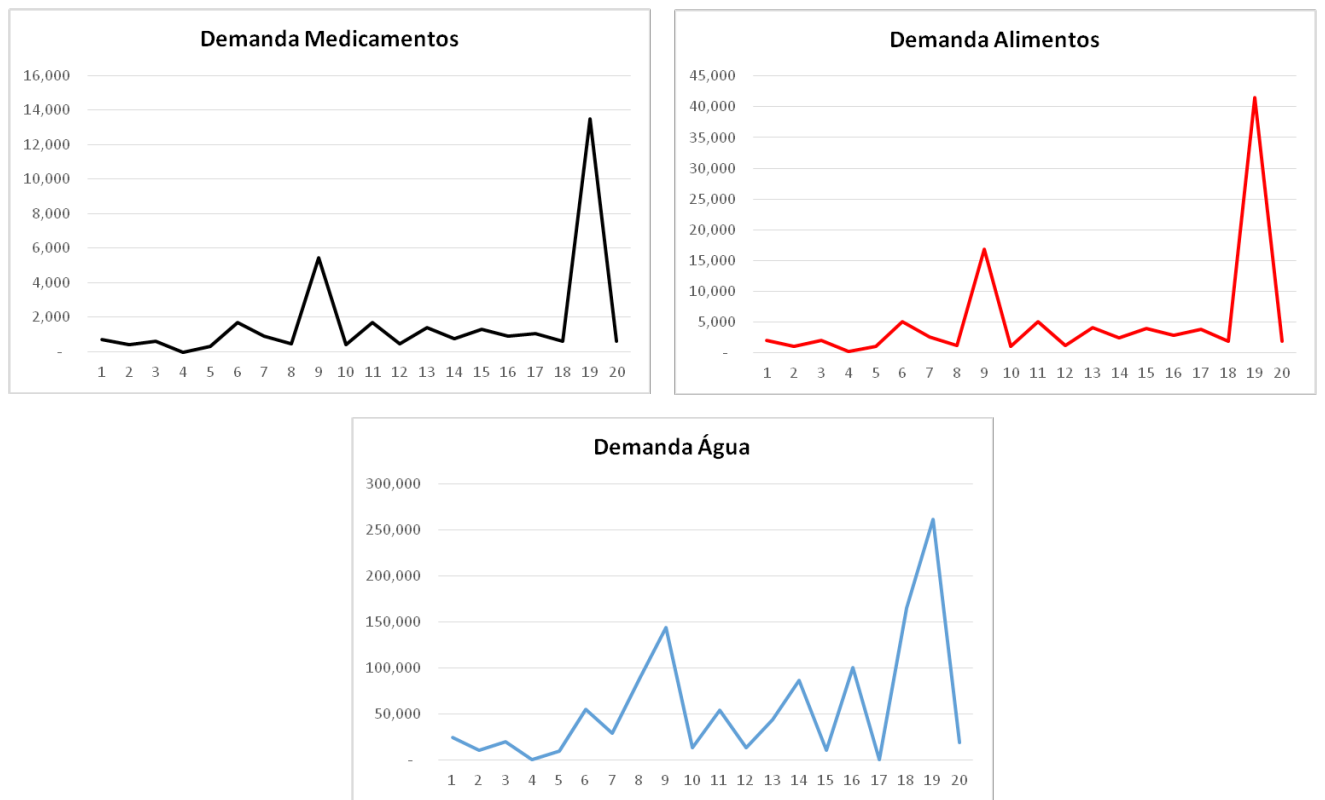

Figura 2: Demanda de KITs de ajuda por dia no desastre região serrana

Fonte: Adaptado Bataglin e Alem (2014) 
Pode-se observar que as séries apresentadas acima (Figura 2) não são estacionárias. Esta característica é constantemente observada em situações de desastres, onde as séries têm uma grande quantidade de não-linearidade e irregularidade - devido à incerteza do evento (informações, necessidades). Para confirmar tal observação foram realizados testes ADF (Augmented Dickey-Fuller), com 5\% de significância, para verificar a estacionariedade das séries apresentadas. A Tabela 2 e a Tabela 3 mostram os resultados dos testes realizados.

Tabela 2: Estatísticas das demandas analisadas

\begin{tabular}{l|ccccc}
\hline \multicolumn{1}{c|}{ Variável } & $\begin{array}{c}\text { Observaçõe } \\
\text { s }\end{array}$ & $\begin{array}{c}\text { Mínim } \\
\text { o }\end{array}$ & $\begin{array}{c}\text { Máxim } \\
\text { o }\end{array}$ & $\begin{array}{c}\text { Médi } \\
\text { a }\end{array}$ & $\begin{array}{c}\text { Desvio } \\
\text { padrão }\end{array}$ \\
\hline Demanda Água & 20 & 0 & 261.00 & 57.36 & 67.339 \\
& & & 0 & 0 & \\
$\begin{array}{l}\text { Demanda } \\
\text { Medicamentos }\end{array}$ & 20 & 0 & 13.500 & 1.658 & 3.010 \\
Demanda Alimentos & 20 & 200 & 41.500 & 5.058 & 9.263 \\
\hline
\end{tabular}

Fonte: Autores (2019)

Tabela 3: Teste Dickey-Fuller

\begin{tabular}{|c|c|c|c|c|}
\hline Variável & $\begin{array}{c}\text { य (Valor } \\
\text { observado) }\end{array}$ & $\begin{array}{l}\tau \text { (Valor } \\
\text { crítico) }\end{array}$ & $\begin{array}{c}\mathrm{p} \text {-valor } \\
\text { (unilateral) }\end{array}$ & $\alpha$ \\
\hline Demanda Água & $-1,786$ & $-0,378$ & 0,614 & $\begin{array}{c}0,0 \\
5\end{array}$ \\
\hline $\begin{array}{l}\text { Demanda } \\
\text { Medicamentos }\end{array}$ & $-1,515$ & $-0,378$ & 0,723 & $\begin{array}{c}0,0 \\
5\end{array}$ \\
\hline Demanda Alimentos & $-1,423$ & $-0,378$ & 0,755 & $\begin{array}{c}0,0 \\
5\end{array}$ \\
\hline
\end{tabular}

Fonte: Autores (2019)

Como o p-valor calculado é maior que o nível de significância alfa=0.05 nos três casos, não se rejeita a hipótese nula H0, portanto a serie não têm estacionariedade. O risco de rejeitar a hipótese nula H0 quando ela é verdadeira é de 61,36\% (Água), 72,35\% (Medicamentos) e 75,54\% (Alimentos).

A partir desta análise fica evidenciado um grande problema na gestão de estoques durante a fase de resposta a um desastre, ou seja, a definição de uma tendência da demanda torna-se muito complexa prejudicando a ação dos gestores que precisam racionalizar os recursos estocados (quantidades não muito elevadas devido ao prazo de validade dos itens, incerteza sobre a ocorrência do desastre).

Descrito incialmente por Corrar e Theóphilo (2004) para gestão de estoques de uma revenda de barracas, o modelo proposto neste artigo foi alterado com o objetivo de atender as necessidades relacionadas a incerteza da demanda e os parâmetros e variáveis relacionados com a LH. A Figura 3 apresenta, de forma simplificada, o modelo proposto. 


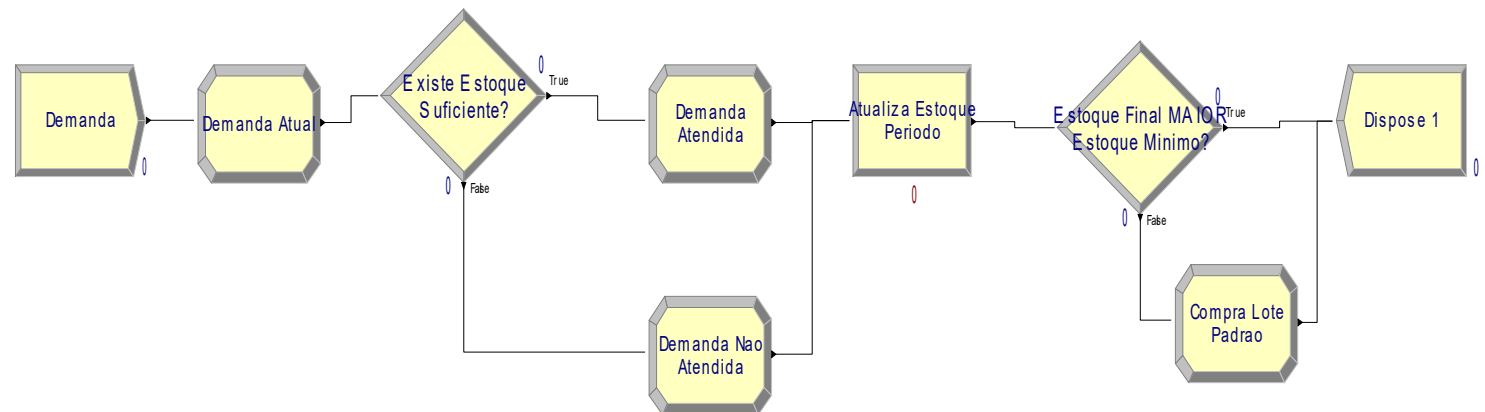

Figura 3: Modelo proposto

Fonte: Autores (2019)

As variáveis do modelo são:

- Produto: Kits de atendimento humanitário (medicamentos, alimentos e água).

- Lote Padrão: Define o tamanho do lote de entrega de cada tipo de kit.

- Leadtime: Define o tempo de entrega, em dias, de cada tipo de kit.

- Estoque Mínimo: Estabelece o nível mínimo de estoque que um determinado kit deve alcançar ao final do período (um dia).

- Demanda: Corresponde ao número de beneficiários (pessoas afetadas pelo desastre) que procuram por kit de ajuda.

- \%Demanda Atendida: Percentual de atendimento da demanda (variável desejada do modelo)

- Variável Aleatória: Simulação da demanda baseado em dados de entrada.

Baseado nos dados originais da demanda (Alimentos, Medicamentos e Água) propostos por Bataglin e Alem (2014) e através do software @Risk, foi possível determinar distribuições de probabilidade que simulam as demandas originais.

Conforme apresentado na Figura 4, para demanda alimentos, o ajuste foi calculado como uma distribuição Log-logistic, para medicamentos e água, como distribuições exponenciais. Portanto, as distribuições de probabilidade apresentadas nesta figura serão números aleatórios a serem utilizados no modelo proposto de forma a representar as demandas de cada um dos kits de ajuda.

\begin{tabular}{|l|l|l|l|l|l|}
\hline Nome & Gráfico & Função & Min & Média & Max \\
\hline ALIMENTOS & RiskLoglogistic(0,2340.8,1.7744, RiskName("ALIMENTOS")) & 0 & 4228.457 \\
\hline MEDICAMENTOS & RiskExpon(1657.5, RiskName("MEDICAMENTOS")) & 0 & $+\infty$ \\
\hline AGUA & RiskExpon(57360,RiskName("AGUA")) & 0 & 1657.5 \\
\hline
\end{tabular}

Figura 4: Variáveis de entrada do modelo

Fonte: Autores (2019)

A Figura 5 representa modelo operacional desenvolvido em planilha EXCEL ${ }^{\circledR}$. Os dados de Estoque Mínimo e Lote Padrão para cada um dos kits estudados, foram sugeridos de acordo com as entrevistas com os especialistas em LH. Na visão dos especialistas, a demanda em caso de desastres é atendida pela união de esforços (doações, governo e organizações não governamentais), portanto, a quantidade dos lotes padrão e estoques mínimos são muito difíceis de serem calculados. Foi sugerida a utilização das quantidades de 
Lote Padrão e Estoque Mínimo como a média da demanda do período estudado.

As etapas para utilização da tabela descrita na Figura 5 são:

i. Escolher o Produto a ser analisado (Alimentos, Medicamentos ou Água);

ii. $\quad$ Variar os dados de entrada (Estoque Mínimo, Lote Padrão e Leadtime);

iii. Executar a simulação através do software @Risk.

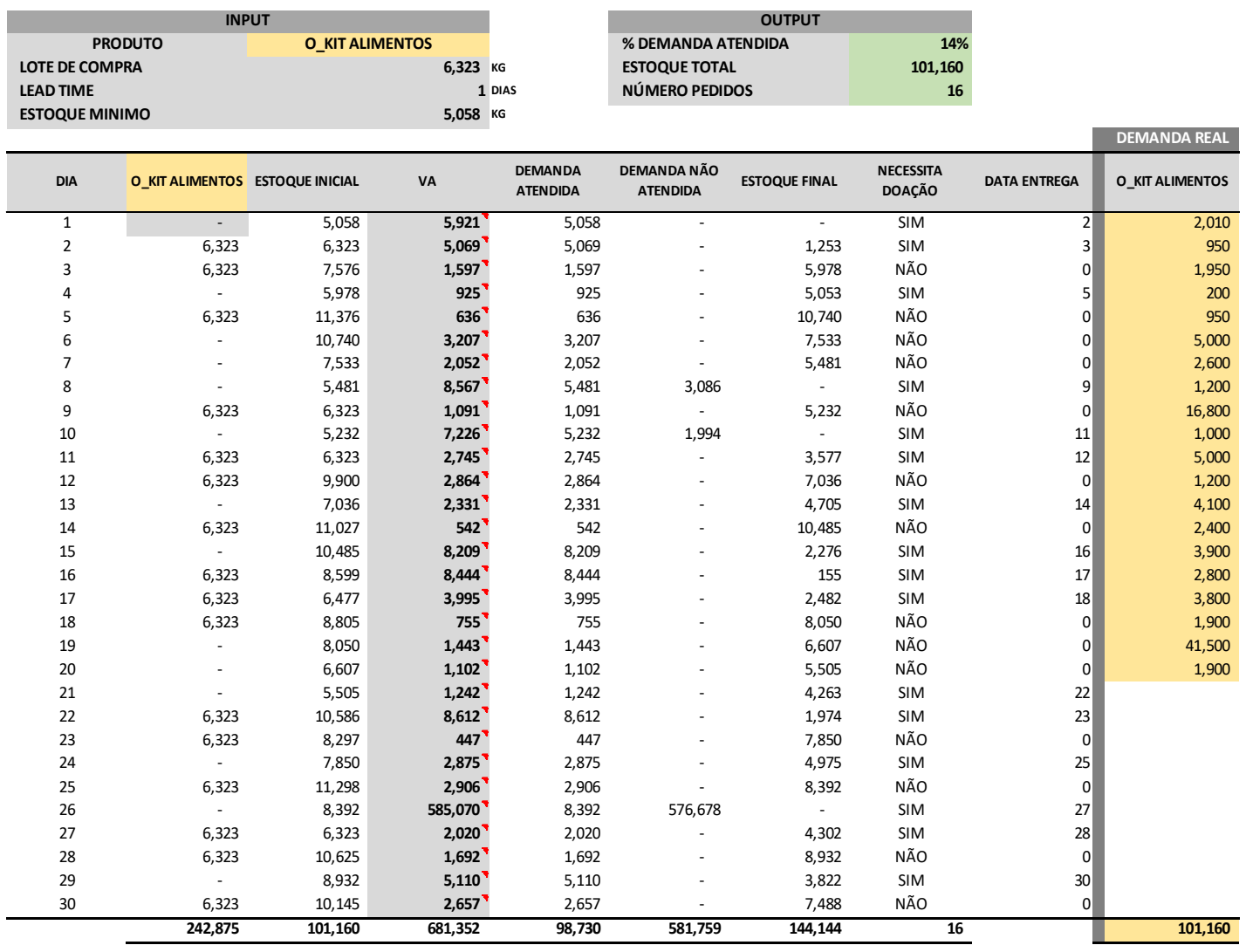

Figura 5: Modelo operacionalizado por planilha eletrônica

Fonte: Autores (2019)

A partir dos dados de entrada do modelo (Demanda original, Lote Padrão, Estoque Mínimo e Leadtime) foram calculados os índices de atendimento da demanda para os três tipos de kits deste estudo. As demandas originais foram substituídas então pelas variáveis aleatórias (criadas a partir da simulação de Monte Carlo). O mesmo procedimento foi replicado 1.000 vezes através do software @Risk, gerando 1.000 prováveis índices de porcentagem atendimento da demanda para cada tipo de demanda.

A Figura 6 apresenta as estatísticas referentes às três demandas estudadas (água, medicamentos e alimentos). No caso dos kits de alimentos foi encontrado um índice médio porcentagem atendimento de atendimento de $77 \%$, para os kits de medicamentos $74 \%$ e para kits de água $74 \%$. 


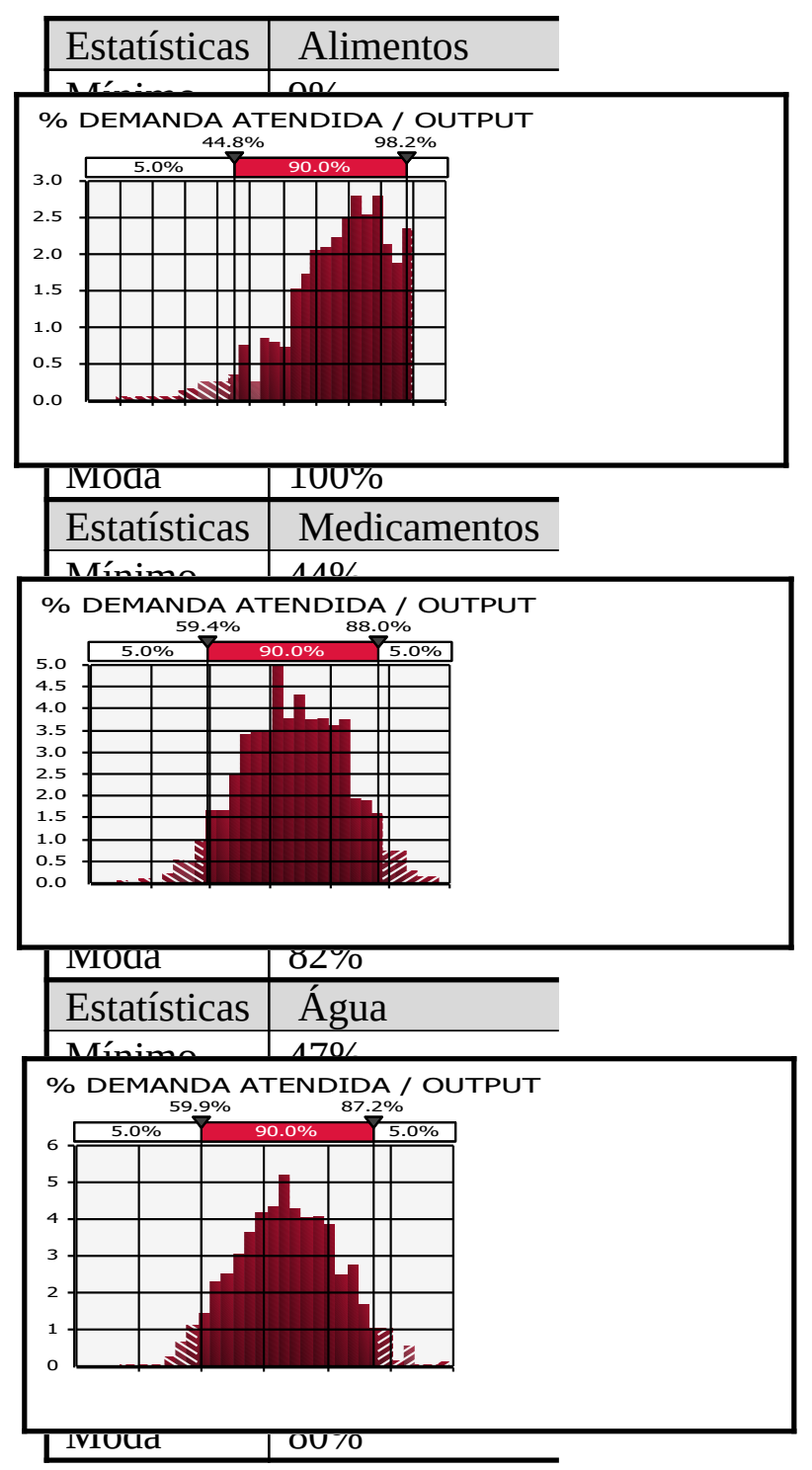

Figura 6: Simulação das demandas utilizando @Risk

Fonte: Autores (2019)

\section{ANALISE DE SENSIBILIDADE}

A partir dos resultados obtidos anteriormente foram realizadas variações no Estoque Mínimo e no Lote Padrão de forma a aumentar a demanda atendida. As simulações foram realizadas para o KIT Alimento onde foram gerados 1.000 índices de porcentagem de atendimento da demanda para cada variação de Estoque Mínimo e Lote Padrão apresentada: $10 \%, 50 \%$ e $100 \%$. No passo seguinte foram realizadas as estatísticas descritivas dos dados gerados através do software @Risk.

A Tabela 4 também inclui o volume total estocado ao longo dos 30 períodos 
estudados - esta medida é importante para verificar os impactos financeiros relacionados ao custo de estocagem.

Tabela 4: Análise de sensibilidade (aumento dos parâmetros de entrada)

\begin{tabular}{|c|c|c|c|c|c|c|c|}
\hline \multirow{2}{*}{$\begin{array}{l}\text { \%Demanda } \\
\text { Atendida }\end{array}$} & \multirow{2}{*}{$\begin{array}{l}\text { Modelo } \\
\text { Atual }\end{array}$} & \multicolumn{3}{|c|}{$\begin{array}{l}\text { Estoque sem alteração } \\
\text { e aumento do Lote Padrão }\end{array}$} & \multicolumn{3}{|c|}{$\begin{array}{l}\text { Aumento do Estoque Mínimo } \\
\text { e do Lote Padrão }\end{array}$} \\
\hline & & $\uparrow 10 \%$ & $\uparrow 50 \%$ & $\uparrow 100 \%$ & $\uparrow 10 \%$ & $\uparrow 50 \%$ & $\uparrow 100 \%$ \\
\hline Mínimo & $9.00 \%$ & $3.33 \%$ & $5.32 \%$ & $12.83 \%$ & $15.23 \%$ & $13.58 \%$ & $5.38 \%$ \\
\hline Máximo & $100.00 \%$ & $100.00 \%$ & $100.00 \%$ & $100.00 \%$ & $100.00 \%$ & $100.00 \%$ & $100.00 \%$ \\
\hline Média & $77.00 \%$ & $77.80 \%$ & $80.11 \%$ & $82.20 \%$ & $78.95 \%$ & $84.57 \%$ & $88.75 \%$ \\
\hline Mediana & $79.00 \%$ & $81.05 \%$ & 83.39\% & $86.14 \%$ & $82.20 \%$ & $88.79 \%$ & $94.66 \%$ \\
\hline Desv Pad & $16.00 \%$ & $16.41 \%$ & $16.14 \%$ & $15.70 \%$ & $16.15 \%$ & $15.97 \%$ & $15.46 \%$ \\
\hline \multirow{2}{*}{$\begin{array}{l}\text { Estoque } \\
\text { Final }\end{array}$} & \multirow{2}{*}{$\begin{array}{l}\text { Modelo } \\
\text { Atual }\end{array}$} & \multicolumn{3}{|c|}{$\begin{array}{l}\text { Estoque sem alteração } \\
\text { e aumento do Lote Padrão }\end{array}$} & \multicolumn{3}{|c|}{$\begin{array}{c}\text { Aumento do Estoque Mínimo } \\
\text { e do Lote Padrão }\end{array}$} \\
\hline & & $\uparrow 10 \%$ & $\uparrow 50 \%$ & $\uparrow 100 \%$ & $\uparrow 10 \%$ & $\uparrow 50 \%$ & $\uparrow 100 \%$ \\
\hline Mínimo & 60,696 & 55,638 & 60,696 & 60,696 & 61,202 & 53,109 & 70,812 \\
\hline Máximo & 121,392 & 133,531 & 136,566 & 151,740 & 139,095 & 144,153 & 161,856 \\
\hline Média & 89,921 & 91,352 & 95,824 & 99,663 & 92,932 & 102,303 & 110,082 \\
\hline Mediana & 91,044 & 89,021 & 98,631 & 101,160 & 94,585 & 98,631 & 111,276 \\
\hline Desv Pad & 11,025 & 11,721 & 13,517 & 15,209 & 11,775 & 14,514 & 18,453 \\
\hline
\end{tabular}

Fonte: Autores (2019)

Ao analisar a Tabela 4, pode ser observada uma melhoria no nível de atendimento da demanda de acordo com aumentos nos Lote Padrão e Estoque Mínimo. A última coluna, por exemplo, mostra que um aumento de $100 \%$ nos Lote Padrão e Estoque Mínimo melhoram o índice de demanda atendida de $77 \%$ para $88.75 \%$, porém aumentam o volume total estocado em 22.4\%. Nota-se, portanto, que aumentos significativos em ambas as variáveis (Lote Padrão e Estoque Mínimo) podem elevar o estoque a cada período e consequentemente podem aumentar os custos operacionais.

A Tabela 5 apresenta uma análise inversa ao resultado apresentado na Tabela 4. Ao diminuir o nível de Estoque Mínimo e aumentar o Lote Padrão, o modelo mostra resultados similares em termos de índice de satisfação, porém o acréscimo de volume estocado é maior (+5.05\%) quando comparado com situações em que o Estoque Mínimo e Lote Padrão são aumentados.

Tabela 5: Análise de sensibilidade (diminuição Estoque Mínimo)

\begin{tabular}{|c|c|c|}
\hline \%Demanda Atendida & $\begin{array}{c}\text { Estoque Mínimo } \downarrow 50 \% \text { e Lote Padrão } \\
\uparrow 100 \%\end{array}$ & $\begin{array}{c}\text { Estoque Mínimo } \downarrow 40 \% \text { e Lote } \\
\text { Padrão } \uparrow 200 \%\end{array}$ \\
\hline $\begin{array}{l}\text { Mínimo - Máximo } \\
\text { Média } \\
\text { Mediana } \\
\text { Desv Pad }\end{array}$ & $\begin{array}{r}9.22 \%-100 \% \\
76.37 \% \\
79.26 \% \\
15.62 \%\end{array}$ & $\begin{array}{r}5.47 \%-100 \% \\
84.44 \% \\
88.64 \% \\
14.97 \%\end{array}$ \\
\hline Estoque Final & $\begin{array}{l}\text { Estoque Mínimo } \downarrow 50 \% \text { e Lote Padrão } \\
\uparrow 100 \%\end{array}$ & $\begin{array}{c}\text { Estoque Mínimo } \downarrow 40 \% \text { e Lote } \\
\text { Padrão } \uparrow 200 \%\end{array}$ \\
\hline $\begin{array}{l}\text { Mínimo - Máximo } \\
\text { Média } \\
\text { Mediana } \\
\text { Desv Pad }\end{array}$ & $\begin{array}{r}60,696-141,624 \\
91,681 \\
91,044 \\
12,710\end{array}$ & $\begin{array}{r}60,696-182,088 \\
107,513 \\
101,160 \\
19,325\end{array}$ \\
\hline
\end{tabular}

Fonte: Autores (2019) 
Nota-se, portanto, que os ajustes referentes aos parâmetros Estoque Mínimo e Lote Padrão geram alterações significativas nos níveis de estoque e atendimento da demanda. Estes, por sua vez, estão diretamente relacionados com a demanda e com fatores externos, não considerados neste estudo, como a capacidade dos armazéns ou custos de estocagem.

Dessa forma, a utilização de um instrumento de controle de estoque que evidencie a disponibilidade de recursos humanitários para atendimento adequado aos beneficiários é essencial para as atividades de gestão de riscos de desastres.

\section{CONSIDERAÇÕES FINAIS}

Devido à incerteza e flutuação da demanda após a ocorrência de um desastre, a gestão dos estoques de emergência é uma atividade de grande importância para evitar a falta e/ou o excesso de recursos. Muitas vezes é preciso utilizar aproximações, pois não se têm registros precisos em termos de quantidade e tipos de itens demandados.

Este artigo, portanto, cumpriu com seu objetivo de propor um modelo de controle de estoques humanitários, usando simulação de Monte Carlo e operacionalizado por planilha Excel de fácil utilização por parte dos stakeholders envolvidos em ações humanitárias. Através dos resultados apresentados, foi possível identificar ajustes no Lote Padrão e Estoque Mínimo de forma a melhorar o índice de atendimento da demanda.

A partir do registro de dados por parte das entidades responsáveis, sugere-se como estudo futuro, a adoção de uma série temporal de demandas para construção de um novo modelo de simulação mais realista e que envolva previsibilidade. Ainda, recomenda-se a aplicação do estudo proposto em um caso real para validar o modelo e ajustá-lo de acordo com as necessidades observadas.

\section{REFERÊNCIAS BIBLIOGRÁFICAS}

[1] AABY, K., HERRMANN, J. W. JORDAN, C. S., TREADWELL, M., WOOD, K. (2006). Montgomery County's Public Health Service Uses Operations Research to Plan Emergency Mass Dispensing and Vaccination Clinics. Informs. Vol. 36, No. 6, pp. 569-579.

[2] BALCIK, B., BOZKIR, C. D. C., KUNDAKCIOGLU, O. E. (2016). A literature review on inventory management in humanitarian supply chains. Surveys in Operations Research and Management Science, 21(2), 101-116.

[3] BANDEIRA, R. A. M., CAMPOS, V. B. BANDEIRA, A. P. F. (2011). Uma visão da logística de atendimento à população atingida por desastre natural. In: Anais do XXV Congresso de Pesquisa e Ensino de Transporte (ANPET).

[4] BATAGLin, L. M. C. e ALEM, D. (2014). O Problema de Localização-Distribuição no megadesastre da Região Serrana no Rio de Janeiro. Gestão da Produção, São Carlos, v. 21, n. 4, p. 865-881.

[5] BARBOSA, E. O., MONTEIRO, V. L., GIANNOTTI, M. A., BRITO Jr., I. (2010). Localização de depósito de suprimentos de alívio para atendimento a desastres naturais no Vale do Paraíba Paulista. In: Anais do XXIV Congresso de Pesquisa e Ensino de Transporte (ANPET).

[6] BEAMON, B. M. e KOTLEBA, S. A. (2006). Inventory management support systems for emergency humanitarian relief operations in South Sudan. International Journal of Logistics Management, Vol. 17 No. 2, pp. 187-212. 
[7] CAMPBELL, A. M. e JONES, P. C. (2011). Prepositioning supplies in preparation for disasters. European Journal of Operational Research 209, 156-165.

[8] ÇELIK, M., ERGUN O., JOHNSON, B., KESHINOCAK, P., LORCA, A., PEKGUN, P. E SWANM, J. (2012) Humanitarian Logistics. Tutorials in Operations Research, INFORMS, Estados Unidos.

[9] CHARLES, A. e LAURAS, M. (2011). An enterprise modelling approach for better optimisation modelling: application to the humanitarian relief chain coordination problem. OR Spectrum, 33(3), pp 815-841.

[10] CHWIF, L. (1999). Redução de modelos de simulação de eventos discretos na sua concepção: uma abordagem causal. $151 \mathrm{f}$. Tese (Doutorado em Engenharia Mecânica) - Escola Politécnica, Universidade de São Paulo, São Paulo.

[11] CORRAR, L. J., THEÓPHILO, C. R. (2004). Pesquisa Operacional para Decisão em Contabilidade e Administração: Contabilometria. São Paulo: Atlas.

[12] COSTA, S. R. A., BANDEIRA, R. A. M., MELOO, L. C. B. B., CAMPOS, V. B. (2014), Humanitarian supply chain: an analysis of response operations to natural disasters. European Journal of Transport and Infrastructure Research 14(3), pp.290310.

[13] ECKHARDT, D. e LEIRAS, A. (2018). A review of required features for a disaster response system on top of a multi-criteria decision: a Brazilian perspective. Production, 28.

[14] GARRIDO, R. A., LAMAS, P., PINO, F. J. (2015). A stochastic programming approach for floods emergency logistics. Transportation research part E: logistics and transportation review, 75, 18-31.

[15] HAN, Y., e WEI, L. (2018). Emergency supply chain management with lateral transshipments and CONWIP. In 2018 7th International Conference on Industrial Technology and Management (ICITM) (pp. 124-129). IEEE.

[16] LEE, E. K., MAHESHWARY, S., MASON J., GLISSON W. (2006). Large-Scale Dispensing for Emergency Response to Bioterrorism and Infectious-Disease Outbreak. Interfaces 36(6):591-607.

[17] LEIRAS, A., BRITO JR., I., BERTAZZO, T. R., PERES, E. Q., YOSHIZAKI, H. T. Y. (2014). Literature review of humanitarian logistics and disaster relief operations research. Journal of Humanitarian Logistics and Supply Chain Management, v.4, n.1.

[18] LODREE JR, E. J. (2011). Pre-storm emergency supplies inventory planning. Journal of Humanitarian Logistics and Supply Chain Management, V. 1 v 1 pp. 50 - 77.

[19] MCCOY, J. H. e BRANDEAU M. L. (2011), Efficient stockpiling and shipping policies for humanitarian relief: UNHCR's inventory challenge. OR Spectrum 33:673-698

[20] MULYONO, N. B. e ISHIDA, Y. (2014). Clustering inventory locations to improve the performance of disaster relief operations. Procedia Computer Science, 35, 13881397.

[21] OZBAY, K. e OZGUVEN, E. (2007). Stochastic humanitarian inventory control model for disaster planning. Transportation Research Board of the National Academies, p. 63-75. 
[22] SPHERE HANDBOOK (2011), Humanitarian Charter and Minimum Standards in Humanitarian Response.

[23] TASKIN, S. e LODREE, E. J. (2010). Inventory decisions for emergency supplies based on hurricane count predictions. International Journal of Production Economics, vol. 126, issue 1 , pages 66-75.

[24] THOMAS, A. e KOPCZAK, L. R. (2005). From logistics to supply chain management: the path forward in the humanitarian sector. Fritz Institute 17.

[25] VAN WASSENHOVE, L.N. (2006). Humanitarian aid logistics: supply chain management in high gear. Journal of Operational Research Society. 57 (5) 475-489.

[26] VERGARA, S. C. (2014). Projetos e relatórios de pesquisa em administração. 15a ed. Rio de Janeiro: Atlas.

[27] TOYASAKI, F., ARIKAN, E., SILBERMAYR, L., FALAGARA SIGALA, I. (2017). Disaster relief inventory management: Horizontal cooperation between humanitarian organizations. Production and Operations Management, 26(6), 1221-1237.

As Referências Bibliográficas devem seguir as normas da ABNT/NBR 6023. 